\title{
A CRITERION FOR NORMALCY
}

\author{
PAUL GAUTHIER
}

\section{Introduction}

Gavrilov [2] has shown that a holomorphic function $f(z)$ in the unit disc $|z|<1$ is normal, in the sense of Lehto and Virtanen [5, p. 86], if and only if $f(z)$ does not possess a sequence of $\rho$-points in the sense of Lange [4]. Gavrilov has also obtained an analagous result for meromorphic functions by introducing the property that a meromorphic function in the unit disc have a sequence of $P$-points. He has shown that a meromorphic function in the unit disc is normal if and only if it does not possess a sequence of $P$-points. In the same paper it was shown that if $\left\{z_{n}\right\}$ is a sequence of $\rho$-points for the function $f(z)$ holomorphic in the unit disc, then $\left\{z_{n}\right\}$ is also a sequence of $P$-points. Moreover if $\left\{z_{n}\right\}$ is a sequence of $P$-points for the holomorphic function $f(z)$, then there is a subsequence of $\left\{z_{n}\right\}$ which is a sequence of $\rho$-points for the function $f(z)$. Thus for holomorphic functions there is a strong relationship between sequences of $\rho$-points and sequences of $P$-points. In this paper we extend the concept of a function possessing a sequence of $\rho$-points so as to be applicable to meromorphic as well as holomorphic functions in the unit disc. It is shown that a sequence $\left\{z_{n}\right\}$ of points of the unit disc is a sequence of $\rho$-points for a meromorphic function $f(z)$ if and only if $\left\{z_{n}\right\}$ is a sequence of $P$-points for $f(z)$. From this equivalence and from Gavrilov's criterion for normalcy quoted above, there follows a new criterion for normalcy. A function $f(z)$ meromorphic in the unit disc is normal if and only if it does not possess a sequence of $\rho$-points. In a subsequent paper this criterion for normalcy will be exploited in studying the distribution of values of meromorphic functions.

Let $z$ and $z^{\prime}$ be two points of the unit disc. We will denote by $\rho\left(z, z^{\prime}\right)$ the hyperbolic non-Euclidean distance between $z$ and $z^{\prime}$. For any two points $a$ and $a^{\prime}$ on the Riemann sphere, we will denote the chordal

Received June 27, 1967.

* This paper is based on the author's doctoral dissertation. 
distance between $a$ and $a^{\prime}$ by $\chi\left(a, a^{\prime}\right)$. A family $F$ of functions defined in the unit disc is said to be equicontinuous at a point $z_{0}$ of the unit disc if for each positive number $r$ there is a positive number $s$ such that

$$
\chi\left(f\left(z_{0}\right), f(z)\right)<r \text {, for } \rho\left(z_{0}, z\right)<s \text { and } f \text { in } F \text {. }
$$

\section{Preliminaries}

Definition 1. Let $f(z)$ be a meromorphic function in the unit disc. A sequence of points $\left\{z_{n}\right\}$ of the unit disc is called a sequence of $P$-points for the function $f(z)$ if for each $r>0$ and each subsequence $\left\{z_{n_{k}}\right\}$ the function $f(z)$ assumes every value, with at most two exceptions, infinitely often in the union of the discs

$$
D_{k}=\left\{z: \rho\left(z, z_{n_{k}}\right)<r\right\}, k=1,2, \cdots .
$$

Theorem 1. Let $f(z)$ be a meromorphic function in the unit disc. A sequence of points $\left\{z_{n}\right\}$ of the unit disc is a sequence of P-points for the function $f(z)$ if and only if there is a sequence of points $\left\{w_{n}\right\}$ of the unit disc and a positive number $r$ such that

$$
\rho\left(z_{n}, w_{n}\right) \rightarrow 0, \text { for } n \rightarrow \infty \text { and } \chi\left(f\left(z_{n}\right), f\left(w_{n}\right)\right)>r, \text { for } n=1,2, \cdots
$$

Proof. Suppose $\left\{z_{n}\right\}$ is a sequence for which there is no corresponding sequence $\left\{w_{n}\right\}$ satisfying (1). Then for any positive number $r$, one can find a sequence of indices

$$
n(1)<n(2)<\cdots<n(k)<\cdots,
$$

such that for all sufficiently large $k$,

$$
\chi\left(f\left(z_{n(k)}\right), \quad f(z)\right)<r, \text { for } \rho\left(z_{n(k)}, z\right)<1 / k .
$$

If in particular we let $r$ be any positive number which is smaller than the diameter of the Riemann sphere, then it can be shown that the subsequence $\left\{z_{n(k)}\right\}$ associated with $r$ has itself a subsequence which is not a sequence of $P$-points. Namely, any subsequence of $\left\{z_{n(k)}\right\}$ whose images under $f(z)$ converge on the Riemann sphere cannot be a sequence of $P$-points. Thus $\left\{z_{n}\right\}$ has a subsequence which is not a sequence of $P$-points. But from the definition it is clear that any subsequence of a sequence of $P$-points is also a sequence of $P$-points. Hence $\left\{z_{n}\right\}$ is not a sequence of $P$-points.

Conversely suppose there is a sequence of points $\left\{w_{n}\right\}$ for which $\rho\left(z_{n}, w_{n}\right)$ 
tends to zero while $\chi\left(f\left(z_{n}\right), f\left(w_{n}\right)\right)$ is bounded away from zero. Let $F=f\left(g_{n}(z)\right)$, where $g_{n}(z)=\left(z+z_{n}\right) /\left(1+\bar{z}_{n} z\right)$. Then clearly the family $F$ of functions. is not equicontinuous at the point 0 . Hence [3, p. 244] for each $r>0, F$ is not a normal family in the set $\{z: \rho(0, z)<r\}$, and so by Montel's theorem [3, p. 248] the family $F$ must assume each value, with at most two exceptions, infinitely often in $\{z: \rho(0, z)<r\}$. That is, $f(z)$ assumes each value of the Riemann sphere, with at most two exceptions, infinitely often in the union of the discs

$$
\left\{z: \rho\left(z_{n}, z\right)<r\right\}, \quad n=1,2, \cdots
$$

Since the same argument can be applied for any positive number $r$ and any subsequence of $\left\{z_{n}\right\}$, it follows that $\left\{z_{n}\right\}$ is a sequence of $P$-points. This concludes the proof.

Lange [4] defined the concept of a sequence of $\rho$-points for a holomorphic function in the unit disc. We will now define what we mean by a sequence of $\rho$-points for a meromorphic function in the unit disc. Definition 2 generalizes that given by Lange in that every sequence of $\rho$-points in the sense of Lange is a sequence of $\rho$-points in the sense of definition 2 . Moreover if a holomorphic function has a sequence $\left\{z_{n}\right\}$ of $\rho$-points in the sense of definition 2 , then $\left\{z_{n}\right\}$ has a subsequence which is a sequence of $\rho$-points in the sense of Lange. These statements are easily verified by comparing the two definitions.

Definition 2. Let $f(z)$ be a meromorphic function in the unit disc. A sequence of points $\left\{z_{n}\right\}$ of the unit disc is called a sequence of $\rho$-points for the function $f(z)$ if there are sequences $\left\{L_{n}\right\}$ and $\left\{r_{n}\right\}$, where

$$
L_{1}>L_{2}>\cdots>L_{n}>\cdots, L_{n} \rightarrow 0 \text {, for } n \rightarrow \infty \text {, }
$$

and

$$
r_{1}>r_{2}>\cdots>r_{n}>\cdots, r_{n} \rightarrow 0 \text {, for } n \rightarrow \infty \text {, }
$$

and there exists a sequence $\left\{D_{n}\right\}$ of open discs,

$$
D_{n}=\left\{z: \rho\left(z_{n}, z\right)<r_{n}\right\},
$$

in the unit disc, having the following property:

(C) in each disc $D_{n}, n=1,2, \cdots$, the function $f(z)$ assumes all values of 
the Riemann sphere with the possible exception of two sets of values $E_{n}$ and $G_{n}$ whose chordal diameters do not exceed $L_{n}$.

TheOREM 2. A sequence of points $\left\{z_{n}\right\}$ of the unit disc is a sequence of o-points for the function $f(z)$ meromorphic in the unit disc if and only if for each $r>0$, there are sets $E(r, n)$ and $G(r, n)$ whose chordal diameters do not exceed $r$, and there is an integer $N(r)$ such that in each disc $\left\{z: \rho\left(z_{n}, z\right)<r\right\}, n>N$, the function $f(z)$ assumes all values of the Riemann sphere with the exception of the sets of values $E(r, n)$ and $G(r, n)$.

Proof. That any sequence of $\rho$-points satisfies the condition stated in the theorem is obvious. Conversely suppose the sequence $\left\{z_{n}\right\}$ satisfies the condition. Then letting $r=1 / m, m=1,2, \cdots$, one obtains sets $E(1 / m, n)$ and $G(1 / m, n), n=1,2, \cdots ; m=1,2, \cdots$. Moreover we may choose the integers $N(1 / m)$ in such a way that

$$
N(1 / 1)<N(1 / 2)<\cdots<N(1 / m)<\cdots
$$

Now we define

$$
r_{n}=1 / m \text { and } L_{n}=1 / m \text {, for } N(1 / m)<n \leq N(1 /(m+1)) .
$$

For $n \leq N(1 / 1)$ we define $r_{n}$ to be 1 and $L_{n}$ to be the diameter of the Riemann sphere. Having defined the sequences $\left\{r_{n}\right\}$ and $\left\{L_{n}\right\}$ we see from definition 2 that the sequence $\left\{z_{n}\right\}$ is a sequence of $\rho$-points for $f(z)$. This concludes the proof.

\section{A Griterion for Normalcy}

We now state our main result.

TheOREm 3. A function $f(z)$ meromorphic in the unit disc is normal if and only if $f(z)$ has no sequence of $\rho$-points.

Proof. This follows immediately from theorem 4 and from Gavrilov's criterion for normalcy which was mentioned in section 1 .

TheOREM 4. A sequence $\left\{z_{n}\right\}$ of points of the unit disc is a sequence of $\rho$-points for a function $f(z)$ meromorphic in the unit disc if and only if the sequence $\left\{z_{n}\right\}$ is a sequence of P-points for the function $f(z)$.

Proof. From theorem 1 it follows easily that any sequence of $\rho$-points 
is a sequence of $P$-points. Conversely suppose $\left\{z_{n}\right\}$ is not a sequence of $\rho$-points. Then there is a positive number $r$ for which the condition in theorem 2 is not satisfied. Hence there is a subsequence, which we again denote by $\left\{z_{n}\right\}$ such that for each $n$ if $D_{n}$ is the non-Euclidean disc with center $z_{n}$ and radius $r$, then the set of values of the Riemann sphere not assumed by $f(z)$ in $D_{n}$ cannot be contained in two sets whose chordal diameters do not exceed $r$. It follows immediately from this that $f(z)$ omits three values $a_{n}, b_{n}$, and $c_{n}$ in $D_{n}$ such that

$$
\chi\left(a_{n}, b_{n}\right) \geq r / 2, \quad \chi\left(a_{n}, c_{n}\right) \geq r / 2, \text { and } \chi\left(b_{n}, c_{n}\right) \geq r / 2 ; n=1,2, \cdots .
$$

From $\left\{z_{n}\right\}$ we may choose a subsequence, which we continue to denote by $\left\{z_{n}\right\}$, such that $\left\{a_{n}\right\},\left\{b_{n}\right\}$, and $\left\{c_{n}\right\}$ converge respectively to (necessarily distinct) values $A, B$, and $C$. Let $f_{n}(z)=f\left(g_{n}(z)\right)$ where $g_{n}(z)$ is a $1-1$ conformal map of the unit disc onto itself such that $g_{n}(0)=z_{n}$. Then for each $n, f_{n}(z)$ omits $a_{n}, b_{n}$, and $c_{n}$ in the disc $\{z: \rho(0, z)<r\}$.

We wish to show that $\left\{f_{n}(z)\right\}$ is a normal family of functions. We may assume that one of the values, say $A$, among $A, B$, and $C$ is not infinite. Set

$$
h_{n}(z)=\left[\left(f_{n}(z)-c_{n}\right) /\left(f_{n}(z)-b_{n}\right)\right] \cdot\left[\left(a_{n}-b_{n}\right) /\left(a_{n}-c_{n}\right)\right] .
$$

Then $\left\{h_{n}(z)\right\}$ omits 0,1 , and $\infty$, and so by Montel's theorem $\left\{h_{n}(z)\right\}$ is a normal family of functions in $\{z: \rho(0, z)<r\}$. Solving for $f_{n}(z)$ in terms of $h_{n}(z)$, we can verify that $\left\{f_{n}(z)\right\}$ is also a normal family. Hence there is a subsequence, which we continue to denote by $\left\{f_{n}(z)\right\}$, which converges spherically uniformly on $\{z: \rho(0, z) \leq r / 2\}$ to a function which is either meromorphic or identically infinite. Since the behavior of $f_{n}(z)$ in $\{z: \rho(0, z) \leq r / 2\}$ is the same as that of $f(z)$ in $\left\{z: \rho\left(z_{n}, z\right) \leq r / 2\right\}$, for each positive number $s$, there is an integer $N$ and a positive number $R$ such that

$$
\chi\left(f\left(z_{n}\right), f(z)\right)<s \text {, for } n>N \text { and } \rho\left(z_{n}, z\right)<R \text {. }
$$

Hence by theorem $1\left\{z_{n}\right\}$ cannot be a sequence of $P$-points. We have shown that any sequence which is not a sequence of $\rho$-points has a subsequence which is not a sequence of $P$-points. But each subsequence of a sequence of $P$-points must also be a sequence of $P$-points, and so any sequence which is not a sequence of $\rho$-points cannot be a sequence of $P$-points. This concludes the proof. 
The author is indebted to Prof. W. Seidel of Wayne State University for his guidance and encouragement.

\section{REFERENCES}

[1] P.M. Gauthier: Sequences of $\rho$-points of meromorphic functions, Amer. Math. Soc. Notices 14 (1967), 257.

[2] V.I. Gavrilov: On the distribution of values of non-normal meromorphic functions in the unit disc (Russian), Mat. Sb. 109 (n.s. 67) (1965), 408-427.

[ 3 ] E. Hille: Analytic function theory, Vol. 2, Boston, 1962.

[4] L.H. Lange: Sur les cercles de remplissage non-Euclidiens, Ann. Sci. École Norm. Sup. (3) 77 (1960), 257-280.

[ 5 ] K. Noshiro: Cluster sets, Berlin, 1960.

[6] A. Ostrowski: Über Folgen analytischer Funktionen und einige Verschärfungen des Picardschen Satzes, Math. Zeitschr. 24 (1926), 215-258.

Wayne State University 